\title{
Cramp fasciculation syndrome in Athletes: A report of two
}

\section{cases}

\author{
Daniel A Rubin*, Jocelyn R Gravlee and Robert L Hatch \\ Department of Community Health and Family Medicine, University of Florida College of Medicine, USA
}

\begin{abstract}
Muscle Cramps are a common chief complaint encountered in sports medicine and primary care clinics. Cramping can be debilitating and significantly impact a person's quality of life and athletic performance. Current theories generally categorize cramps as those caused by overexertion of a specific muscle group or due to sodium deficiencies. In patients that have associated fasciculations, the diagnosis of Cramp Fasciculation Syndrome (CFS) should be considered as the pathophysiology and treatment is different. Cramp Fasciculation syndrome (CFS) is a chronic, benign condition characterized by fasciculations and significant muscle cramps. The cramps are typically triggered by exertion and are due to peripheral nerve hyper-excitability rather than muscle fatigue or electrolyte deficiency. Examination is typically normal with no evidence of motor weakness or atrophy. Anticonvulsants such as Gabepentin has been shown to reduce cramping.
\end{abstract}

\section{Introduction}

Cramp Fasciculation syndrome (CFS) is a chronic, benign condition characterized by fasciculations and significant muscle cramps [1]. The cramps are typically triggered by exertion and are due to muscle hyperexcitability [2] rather than fatigue or electrolyte deficiency. Despite the fact that CFS is well described in the neurology literature and can impair athletic performance, we found no mention of CFS in the sports medicine literature. We report two cases that demonstrate classic manifestations of CFS and the impact it may have on athletes' lives. The cause of each athlete's cramping went undiagnosed for many years, despite consultations with generalist physicians, trainers and sports medicine specialists.

\section{Case 1}

A 51-year-old triathlete presented with a 20-year history of cramping. Cramps were precipitated by prolonged hard exertion, brisk motions and placing muscles in a shortened position after working. During swims, even gentle push offs from the wall caused foot and calf cramping, and he developed a technique of slow, awkward turns to avoid cramps. Plantar foot cramps occurred after swimming just 20 meters with flippers but could be prevented by modifying flippers to hold toes in 45 degrees of dorsiflexion. He had completed a full iron distance race, but hamstring cramping forced him to reduce his bike and run pace well below potential in any race lasting over 90 minutes. Vigorous hydration during exercise (sports drinks supplemented with additional $\mathrm{NaCl}$ and $\mathrm{KCl}$ ) and magnesium oxide $1 \mathrm{~g}$ daily provided mild but noticeable benefit during longer workouts, but no benefit during swims. After heavy workouts, he often woke 2-5 times per night with cramps in the feet, calves, and thighs. Fasiculations were common in his soleus and gastrocnemius. These were first noted at age 28. No other neuromuscular complaints were noted. Past medical history included occasional nocturnal leg cramps during childhood and exercise induced asthma. Medications included albuterol MDI before winter workouts. There was no family history of neuromuscular disorders. Apart from fasciculations, physical exam was normal, including muscle bulk, strength, sensation, pulses, and reflexes. Electrolytes, complete blood count, liver function tests, and urinalysis were all normal. Gabapentin at doses up to $1200 \mathrm{mg}$ per day did not reduce cramping, and side effects (disequilibrium) prevented higher dosing.

\section{Case 2}

A 57-year-old man presented with a 40-year history of cramping and bilateral calf fasciculations, first noted in high school while competing in sprinting events. A variety of activities including farm work, rollerblading, white water rafting, running and horseback riding provoked severe cramping of the upper and lower extremities throughout his life. Severe nocturnal cramping in the back and extremities was common after heavy exertion, especially if associated with underhydration. Aggressive hydration with electrolyte drinks was minimally helpful. Empiric trials of magnesium, potassium, and L- carnitine did not alleviate symptoms. No other neuromuscular complaints were noted. There was no myalgia, atrophy or loss of strength. Past medical history was remarkable for mild carpo/metacarpal joint osteoarthritis. There was no family history of neuromuscular conditions. His physical examination was normal, including strength, reflexes, muscle bulk, cranial nerves, and sensation. Laboratory evaluation yielded a creatine kinase (CK) of $600 \mathrm{U} / \mathrm{L}$. Repeat CK at a time of less cramping yielded a near-normal value. Complete blood count, electrolytes, liver function tests, rheumatoid factor, TSH, ANA, erythrocyte sedimentation rate, and urinalysis were all normal. EMGs were normal. A trial of Gabepentin $300 \mathrm{mg}$ four times a day significantly reduced cramping and fasciculations, but daytime drowsiness forced a reduction in dose and effectiveness. The patient then gave up running.

${ }^{*}$ Correspondence to: Daniel Rubin, Department of Community Health and Family Medicine, University of Florida College of Medicine, Family Medicine at Haile Plantation, 2846 SW 87th Way Gainesville, FL 32608, USA. Tel: (352) 265-0944; E-mail: rubind@ufl.edu

Key words: cramp fasciculation syndrome, exercise, muscle cramps

Received: September 11, 2019; Accepted: September 17, 2019; Published: September 20, 2019 
After a more gradual upward titration, he was able to tolerate higher doses with much better control of cramping, especially the nocturnal cramping which was significantly ameliorated.

\section{Discussion}

Denny-Brown and Foley first described cramps in association with benign fasciculations in 1948 [3]. In 1991, Tahmoush et al. coined the term "cramp-fasciculation syndrome" [4]. They described nine patients with muscle aching, cramps, stiffness, exercise intolerance, and peripheral nerve hyperexcitability. Patients with CFS have a completely normal neurologic examination other than fasciculations, generally in the lower extremities. Electromyograms may be normal or may reveal frequent fasciculations. Repetitive nerve stimulation is a clinically useful test for the diagnosis of CFS. In one study, $81 \%$ of patients who met clinical criteria for CFS demonstrated abnormal nerve hyperexcitability [2]. Serum antibodies to voltage-gated potassium channels have been found in some patients with CFS [5]. Testing for antibodies to potassium channels is not recommended because no targeted treatment is available. Creatine kinase may be elevated in patients with CFS, but this finding is neither diagnostic nor specific [4]. Muscle biopsy is typically normal but may show mild neurogenic changes [4].

Treatment of CFS can be challenging. Since the underlying cause involves peripheral nerve hyperexcitablity, anticonvulsants should be effective. Indeed, Gabapentin has been shown to reduce cramping [6]. Unfortunately, as case 2 illustrates, side effects can limit effective treatment. Carbamazepine and phenytoin can reduce nerve hyperexcitability and may also be considered [7]. Although Quinine was commonly used to treat cramps, it is no longer recommended due to clear risks and no proven benefits [8]. We were unable to find any studies of non-pharmacologic treatment of CFS, such as electrolyte supplementation or stretching.

Despite the significant limitations CFS can impose, the condition is considered benign. Cramps and fasciculations have been noted in motor neuron diseases such as amyotrophic lateral sclerosis [7]. One case report documented such findings preceding motor neuron death in a motor neuron disease [9]. When evaluating a patient with possible CFS, a careful examination for any motor weakness or atrophy should be performed and documented. If noted, other neuromuscular conditions should be considered. Cramping related to electrolyte deficiency should be considered, and supplemental $\mathrm{NaCl}$ can be added to sports drinks if sodium deficiency is suspected [10]. Referral to a neurologist could be considered if symptoms are severe, treatment results unsatisfactory, or the diagnosis is in question.

Cramp Fasciculation Syndrome is a distinct clinical entity that can impact an athlete's performance and quality of life. Although CFS is not common, it is probably underappreciated in the sports-medicine community and should always be considered in unusual or refractoryto-treatment cramping disorders. Specific treatment is available and is worth considering in certain cases.

\section{References}

1. de Entrambasaguas M, Ortega-Albás JJ, Martínez-Lozano MD, Díaz JR (2006) Bronchial involvement in the cramp-fasciculation syndrome. Eur Neurol 56: 124-126. [Crossref]

2. Harrison TB, Benatar M (2007) Accuracy of repetitive nerve stimulation for diagnosis of the cramp-fasciculation syndrome. Muscle Nerve 35: 776-780. [Crossref]

3. Denny-Brown D, Foley J (1948) Myokimia and the benign fasciculation of muscle cramps. Trans Assoc Am Phys 61: 88-96.

4. Tahmoush AJ, Alonso RJ, Tahmoush GP, Heiman-Patterson TD (1991) Crampfasciculation syndrome: a treatable hyperexcitable peripheral nerve disorder. Neurology 41: 1021-1024. [Crossref]

5. Newsom-Davis J (2005) Neuromuscular junction channelopathies: a brief overview. Acta Neurologica Belgica 105: 181-186. [Crossref]

6. Serrao M, Rossi P, Cardinali P, Valente G, Parisi L, et al. (2000) Gabapentin treatment for muscle cramps: an open-label trial. Clin Neuropharmacol 23: 45-49. [Crossref]

7. Miller TM, Layzer RB (2005) Muscle cramps. Muscle Nerve 32: 431-442.

8. Food and Drug Administration (2006) Drug products containing quinine; enforcement action dates. Federal Register 71: 75557-75560.

9. de Carvalho M, Swash M (2004) Cramps, muscle pain, and fasciculations: not always benign? Neurology 63: 721-723. [Crossref]

10. Bergeron M (2008) Muscle cramps during exercise: is it fatigue or electrolyte deficit? Curr Sports Med Rep 7: S50.

Copyright: (C2019 Rubin DA. This is an open-access article distributed under the terms of the Creative Commons Attribution License, which permits unrestricted use, distribution, and reproduction in any medium, provided the original author and source are credited. 